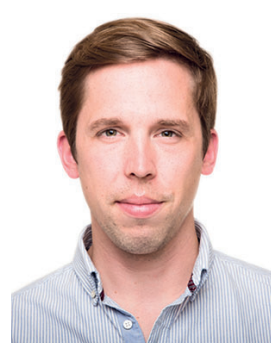

Matthias Raspe

Koordination «PneumoCampus»

\section{Atemnot, Therapiemaßnahmen und Problemlösungen}

Ein Patient mit akutem Atemnotsyndrom, dessen Röntgen- und CT-Thorax deutliche Auffälligkeiten zeigen, beschäftigt die Blickdiagnosen dieser Ausgabe. Ob Sie mit Ihrer Diagnose richtig liegen, können Sie wie immer auf der KARGER-KOMPASS-PNEUMOLOGIE-Webseite überprüfen.

Wie angekündigt setzen wir unsere kleine Serie zu den aktuellen Therapiemaßnahmen beim nicht kleinzelligen Lungenkarzinom (NSCLC) fort - in diesem Heft widmet sich der Steckbrief dem Tumorstadium III.

Wer Ihre erste Anlaufstelle bei Problemen in der Weiterbildung sein könnte, verrät Ihnen ein neuer «Überlebenstipp für die Weiterbildung».

\section{Blickdiagnosen \\ Welche Diagnose vermuten Sie?}

Der 20-jährige Patient wurde zur Therapie eines akuten Atemnotsyndroms (acute respiratory distress syndrome, ARDS) aus einem externen Krankenhaus in unsere Klinik übernommen. Der Patient hatte sich dort über die Rettungsstelle 2 Tage vorher mit Husten und Hämoptysen seit wenigen Tagen vorgestellt - diese Symptome waren erst kurz zuvor aufgetreten. Außerdem bestanden atemabhängige thorakale Schmerzen, Fieber von knapp $39^{\circ} \mathrm{C}$ und eine progrediente Belastungsdyspnoe. Der Patient war bis zur Aufnahme aktiver Raucher mit einer Packung Zigaretten am Tag (kumulativ etwa 2 Packungsjahre). Im Verlauf des Klinikaufenthaltes wurde bekannt, dass der Patient bereits ein Jahr zuvor mit einer ähnlichen, aber nicht so ausgeprägten Symptomatik stationär behandelt worden war (CT-Thorax-Befunde A1 und 2). In die- sem Rahmen wurde auch eine offene Lungenbiopsie durchgeführt. Laborchemisch fiel aktuell eine ausgeprägte Eisenmangelanämie mit einem Hb-Wert von initial 5,5g/dl auf (Referenzwert: 13,5-17). Eine Röntgen- (B) und eine Computertomographie (C) des Thorax zeigten deutliche Auffälligkeiten. Eine breite kalkulierte antibiotische Therapie wurde eingeleitet. Der Patient wurde intubiert und beatmet übernommen. Es schloss sich ein komplizierter intensivmedizinischer Aufenthalt an.

\section{Auflösung unter: \\ www.karger.com/blickdiagnose-kkp-4-2019}

Kontaktadresse: Dr. Matthias Raspe, Medizinische Klinik mit Schwerpunkt Infektiologie und Pneumologie, Charité - Universitätsmedizin Berlin, Augustenburger Platz 1, 13353 Berlin, Deutschland, matthias. raspe@charite.de
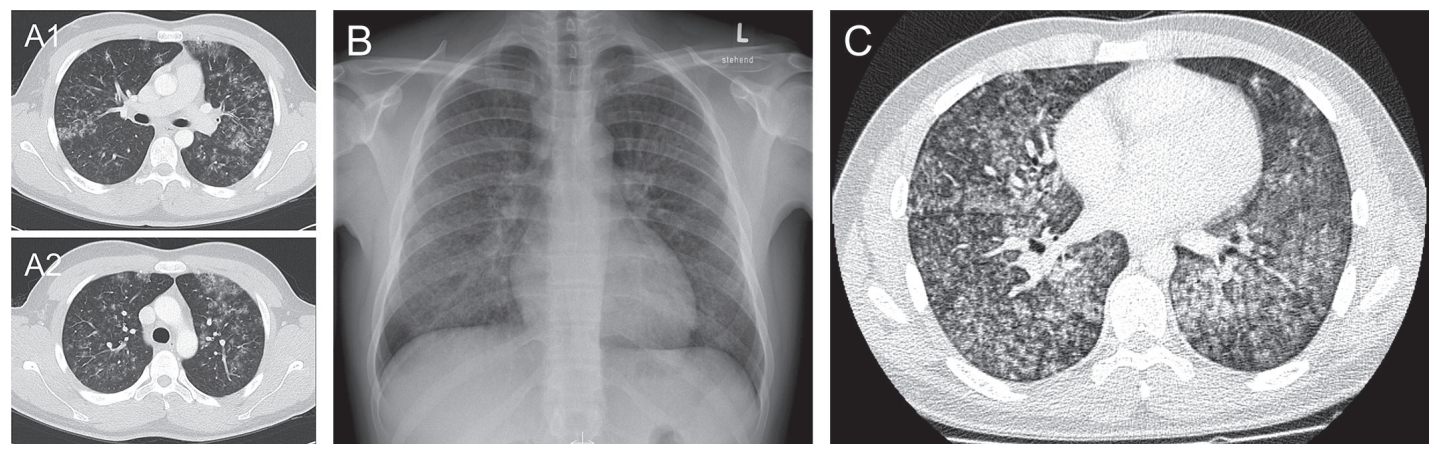

Bildbefunde mit freundlicher Genehmigung von PD Dr. med. C. Althoff, Leitung Interventionsradiologie Charité Campus Mitte, Institut und Klinik für Radiologie CCM und CVK, Charité - Universitätsmedizin Berlin.

\title{
KARGER
}

(C) 2019 S. Karger GmbH, Freiburg 


\section{Steckbriefe zur aktuellen Therapie des nicht kleinzelligen Lungenkarzinoms Stadium III}

Das Lungenkarzinom ist bei Frauen der dritt- und bei Männern der zweithäufigste maligne Tumor in den deutschsprachigen Ländern mit einer hohen tumorspezifischen Mortalität (bei Männern Platz 1 und bei Frauen Platz 3 in Deutschland). Erfreulicherweise haben sich im letzten Jahrzehnt Therapieoptionen und Prognose durch Zulassung vieler neuer Präparate aus den Bereichen Tyrosinkinaseinhibitoren und Immuntherapie deutlich verbessert - hiervon profitieren im Wesentlichen Patienten in fortgeschrittenen Krankheitsstadien.

In pneumologischen Abteilungen sind Patienten mit Lungenkarzinomen häufig. Andererseits erfordert die Begleitung dieser Patienten viel Erfahrung und Wissen um den aktuellen State-of-the-art in Diagnostik und Therapie. Gerade jüngere Kolleginnen und Kollegen werden hier vor eine große Aufgabe gestellt. Mit dieser 4-teiligen Serie wollen wir einen praxisnahen und übersichtlichen Beitrag zur aktuellen Therapie des nicht kleinzelligen Lungenkarzinoms (non-small cell lung carcinoma, NSCLC) geben. Im zweiten Teil soll es um die Therapie des NSCLC im heterogenen Stadium III gehen.

Die Klassifikation des NSCLC erfolgt aktuell nach IASLC (International Association for the Study of Lung Cancer)/UICC (Union for International Cancer Control-Version 8 [1]. Die folgende Tabelle gibt einen Überblick über die Einteilung des Stadiums III.

Tumorstadium III des NSCLC nach IASLC/ UICC Version 8

\begin{tabular}{llll}
\hline Stadium & Primärtumor & Lymphknoten & Fernmetastasen \\
\hline IIIA & T1a-c & N2 & M0 \\
& T2a-b & N1 & \\
& T3 & N0 \\
& T4 & N1 \\
\hline T4 & N3 \\
& T1a-b & N2 $a-b$ & \\
& T3 & N3 \\
\hline IIIC & T4 & & \\
& T4 &
\end{tabular}

Das Stadium III ist das heterogenste Tumorstadium innerhalb aller NSCLC-Stadien und reicht von lokal noch relativ begrenzten bis hin zu weit fortgeschrittenen Tumorsituationen (siehe Tab. oben). Dementsprechend sind die 5-Jahres-Überlebensraten ebenfalls sehr unterschiedlich: Für das Substadium IIIA liegen sie bei 41\%, für IIIB bei 24\% und für IIIC bei nur noch 12\%. Als Stagingmaßnahmen sind immer eine PET-CT (Positronenemissionstomographie/Computertomographie) und CMRT (Cranio-Magnetresonanztomographie) notwendig. Gegebenenfalls sollte die Evaluation der funktionellen Operabilität erfolgen. Zusätzlich muss eine invasive (in der Regel EBUS (endobronchialer Ultraschall)-gestützte) Abklärung aller suspekten (in der Regel FDG (Fluordesoxyglukose)-aviden) Lymphknotenstationen durchgeführt werden. Die grundsätzliche
Entscheidung ist, ob ein noch operables Stadium III vorliegt. Dies ist nicht einheitlich und abschließend definiert. Wesentliches Entscheidungskriterium für Operabilität ist, dass eine hohe RO-Wahrscheinlichkeit gegeben sein muss (z.B. bei T3N1-Stadien oder einzelnen N2- oder einer "begrenzten“ Anzahl kleiner N2-Lymphknoten ohne kapselüberschreitendes Wachstum). Wird der Patient operiert, schließen sich in der Regel eine adjuvante Chemotherapie und gegebenenfalls eine Strahlentherapie bei N2- (ab N2a2, folgende Tab.) oder R1-Situationen an.

Subklassifikation des NSCLC-Stadiums III nach Robinson [2]

\begin{tabular}{|c|c|}
\hline Stadium & Beschreibung \\
\hline$\| A_{1}$ & $\begin{array}{l}\text { Inzidentelle Lymphknotenmetastasen nach postoperativer } \\
\text { Aufarbeitung im Präparat }\end{array}$ \\
\hline$\| \mathrm{A}_{2}$ & $\begin{array}{l}\text { Intraoperativer Nachweis von Lymphknotenmetastasen } \\
\text { in einer Lymphknotenstation }\end{array}$ \\
\hline$\| A_{3}$ & $\begin{array}{l}\text { Präoperativer Nachweis von Lymphknotenmetastasen in } \\
\text { einer oder mehreren Lymphknotenstationen (sogenannte } \\
\text { uni- oder multilevel) } \\
\text { Nach IASLC/ UICC } 8 \text { wird hier noch unterschieden: } \\
\text { N2a1 = «skip lesion» single level N2 (N1-Stationen werden } \\
\text { übersprungen) } \\
\text { N2a2 = N1 und single level N2 befallen } \\
\text { N2b = mehrere N2-Stationen befallen }\end{array}$ \\
\hline$\| \mathrm{A}_{4}$ & $\begin{array}{l}\text { Ausgedehnte («bulky») oder fixierte N2-Metastasen } \\
\text { oder Metastasen in mehreren Lymphknotenstationen } \\
\text { (mediastinale Lymphknoten }>2-3 \mathrm{~cm} \text { ) mit extrakapsulärer } \\
\text { Infiltration; Befall mehrerer N2-Lymphknotenpositionen; } \\
\text { Gruppen multipler befallener kleinerer (1-2 cm) } \\
\text { Lymphknoten }\end{array}$ \\
\hline
\end{tabular}

In primär nicht operablen Situationen kommen eine primäre Radiochemotherapie oder verschiedene kombinierte Therapiekonzepte aus Chemotherapie, Radiotherapie und Operation in Frage. Mit der jüngeren Zulassung des Immuntherapeutikums Durvalumab als adjuvante Therapie nach Radiochemotherapie haben sich die therapeutischen Möglichkeiten im Stadium III nochmals erweitert. Die Abbildung auf der nächsten Seite zeigt den hausinternen Therapiealgorithmus der Charité - Universitätsmedizin Berlin für das Stadium III.

\section{Potenziell operable Stadien III (cT3-4N0-1 und T1-3N2)}

Sind funktionelle und technische Operabilität gegeben, entscheidet sich das weitere Vorgehen nach dem Lymphknotenstaging (PET-CT, EBUS). Bei einem Lymphknotenbefall bis Maximal-singlelevel N2 (maximal N2a2) wird eine primäre Operation ((VATS (video assisted thoracoscopic surgery)-)Lobektomie und Lymphadenektomie (LAD)) angestrebt, gefolgt von 4 Zyklen adjuvanter Chemotherapie (für Protokolle siehe Teil 1 dieser Serie zu den NSCLC-Stadien I/II in Heft 3/2019 von KARgER KompasS PNEUMOLOGIE). Besteht eine Multilevel-N2-Situation (IIIA3) mit Aussicht eine operable Situation zu erreichen, favorisieren wir 4 Zyklen einer neoadjuvanten 


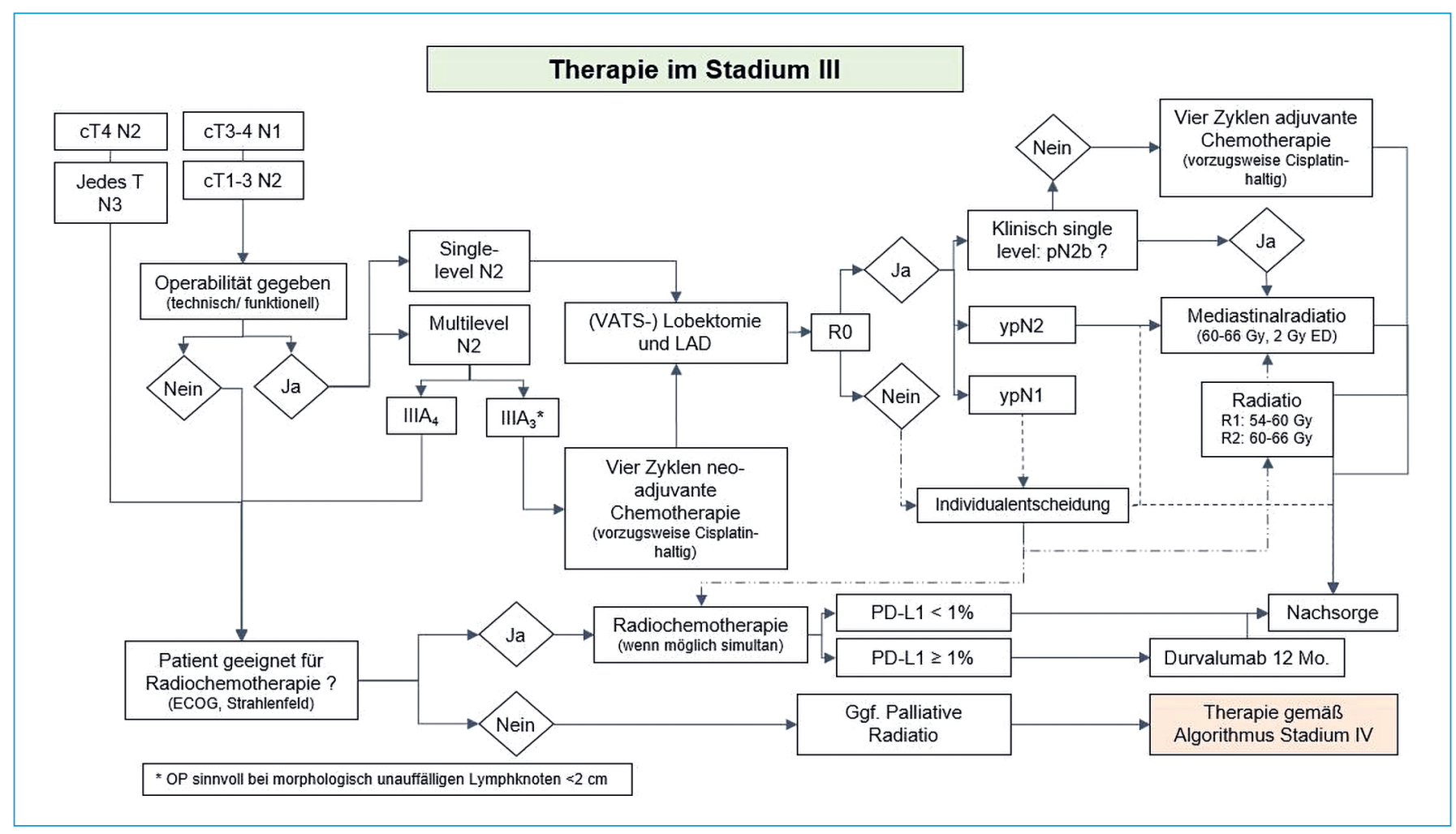

(Hausinterner) Therapiealgorithmus des NSCLC im Stadium III. LAD, systematische Lymphonodektomie; VATS, videoassistierte Thorakoskopie; ED, Einzeldosis. @ Nikolaj Frost 2019.

Chemotherapie, gefolgt von einer Operation und anschließender Mediastinalbestrahlung. Bei R1- oder R2-Situationen ist eine zusätzliche Bestrahlung der Absetzungsebene vorgesehen. Eine ausgedehnte N2-Metastasierung (IIIA4) bei den oben genannten Tumorstadien sollte mit einer Radiochemotherapie behandelt werden.

\section{Nicht operable Stadien III (cT4N2 und jedes TN3)}

In diesen Tumorstadien ist die kombinierte simultane Radiochemotherapie das Mittel der Wahl. Bei Patienten mit eingeschränktem Performance-Status der Eastern Cooperative Oncology Group (ECOG/PS) oder bei nicht möglicher Bestrahlung, z.B. aufgrund eines zu großen Strahlenfeldes, bleiben individuelle Therapiekonzepte bzw. Therapien nach Stadium IV des NSCLC. Patienten sollte nach erfolgter simultaner Radiochemotherapie im Stadium III bei einer PD-L1-Expression n1\% die 12-monatige adjuvante Immuntherapie mit Durvalumab angeboten werden.

In der verblindeten und randomisierten Phase 3-PACIFIC-Studie [3] erhielten von 709 Patienten mit einem NSCLC im nicht-resezierbaren Stadium IIIA ohne Tumorprogress nach einer Radiochemotherapie 473 Patienten eine konsolidierende Immuntherapie mit dem
PD-L1-Antikörper Durvalumab für 12 Monate (2:1-Randomisierung; alle 2 Wochen 10mg/kg Körpergewicht i.v.; 236 Patienten erhielten Placebo). Als primärer Endpunkt lag das progressionsfreie Überleben (PFS) im Studienzeitraum bei 16,8 Monaten im Interventionsund bei 5,6 Monaten im Placebo-Arm (HR 0,52; 95\% KI 0,42 bis 0,65; $\mathrm{P}<0,001)$. Grad 3/4-Toxizitäten wurden bei 29,9\% der Patienten im Interventions- und bei 26,1\% der Patienten im Placebo-Arm beobachtet. Durch die EMA ist die Zulassung auf Patienten mit PD-L1Expression $\geq 1 \%$ begrenzt. In der PACIFIC-Studie bestand diese Begrenzung nicht.

\section{Literatur}

1 Goldstraw P, Chansky K, Crowley J, et al.: The IASLC Lung Cancer Staging Project: Proposals for Revision of the TNM Stage Groupings in the Forthcoming (Eighth) Edition of the TNM Classification for Lung Cancer. J Thorac Oncol 2016;11:39-51.

2 Robinson LA, Ruckdeschel JC, Wagner H Jr., Stevens CW, American College of Chest P: Treatment of non-small cell lung cancer-stage IIIA: ACCP evidence-based clinical practice guidelines (2nd edition). Chest 2007;132:243S-65S.

3 Antonia SJ, Villegas A, Daniel D, et al.: Durvalumab after Chemoradiotherapy in Stage III Non-Small-Cell Lung Cancer. N Engl J Med 2017;377:1919-1929. 
Überlebenstipp für die Weiterbildung

«Bei größeren und unüberbrückbaren Problemen mit der Weiterbildung kann der sogenannte Ombudsmann bzw. die Ombudsfrau weiterhelfen und vermitteln. Die Ombudsperson nimmt ihre Aufgaben im Ehrenamt wahr und ist von der jeweiligen Landesärztekammer (nicht alle Kammern haben eine Person in dieser Funktion) beauftragt. Thre Arbeit ist vertraulich und Maßnahmen erfolgen in enger Absprache mit dem Ratsuchenden.»

Matthias Raspe 